\title{
Magnetic Imaging on the Nanometer Scale Using Low-Temperature Scanning Probe Techniques
}

\author{
M. Zech, ${ }^{\star}$ C. Boedefeld, F. Otto, and D. Andres
}

attocube systems AG, Koeniginstrasse 11a RGB, Munich, Germany

^martin.zech@attocube.com

\section{Introduction}

Multiple techniques now exist for the investigation of nanoscale magnetic properties, extending from Lorentz microscopy [1] and magneto-optical imaging (MOKE) [2] to scanning probe microscopy approaches (see Figure 1 for an overview). Among the latter, the most widely used techniques offering both high spatial and high magnetic-field resolution are magnetic force microscopy (MFM) [3] and scanning Hall probe microscopy (SHPM) [4]. Both techniques are well known for their versatility and ease of use and can be further adapted for operation in cryogenic conditions. This property is crucial for all areas of research where high magnetic fields are required and where the influence of thermal energy/broadening needs to be suppressed. For example, much of today's fundamental research on superconductivity [5], spintronics [6], and magnetic data storage [7] is taking place at low temperatures.

The MFM and SHPM techniques are complementary in the sense that SHPM provides the user with non-invasive, quantitative measurements of the local magnetic field, whereas MFM is sensitive to the gradient of the local force but with almost one order of magnitude higher spatial resolution. With its attoMFM and attoSHPM products, attocube systems addresses both of these techniques, allowing the researcher to investigate magnetic properties with high spatial resolution and sensitivity in environments ranging from ultra-low

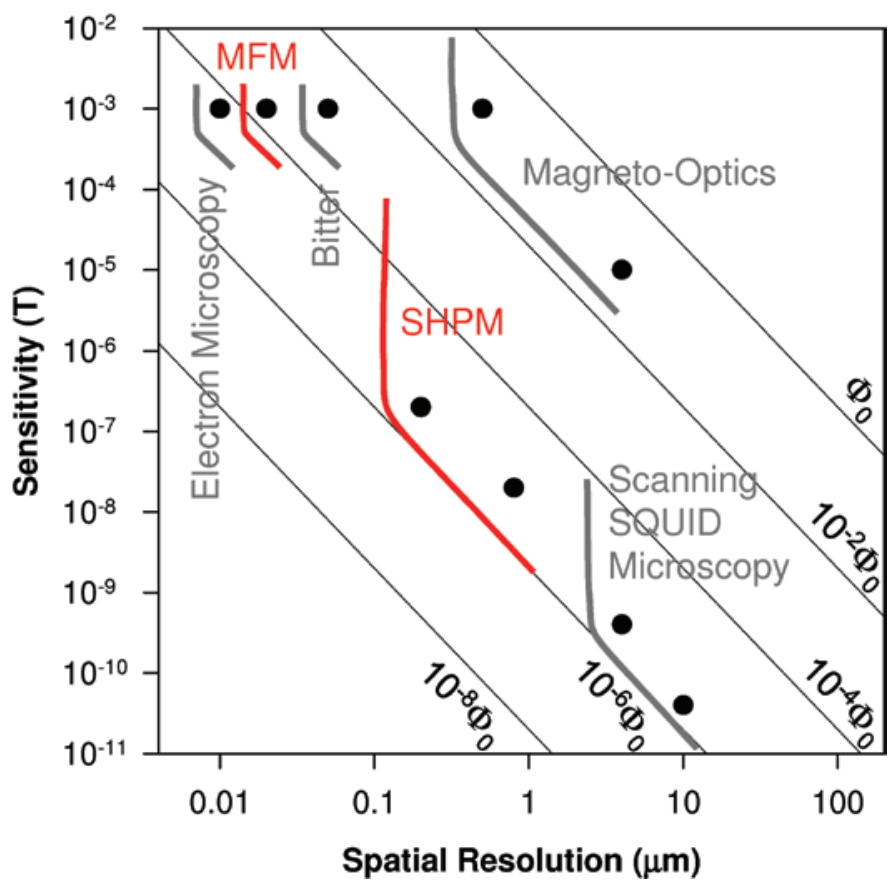

Figure 1: Magnetic field sensitivity as a function of spatial resolution, demonstrated for different magnetic imaging techniques [9]. temperature (down to the $\mathrm{mK}$ regime) and high magnetic fields (up to $15 \mathrm{~T}$ ) to ambient conditions (see Figure 2).

\section{Magnetic Force Microscopy}

Magnetic force microscopy (MFM) [3] is a technique derived from atomic force microscopy (AFM) [8], in which an etched silicon cantilever/tip combined with optical deflection detection is used to precisely measure local forces such as those caused by van der Waals or Coulomb interaction. MFM takes advantage of cantilevers with very low spring constant $K$ and tips with magnetic coatings, typically $\mathrm{NiCr}$ or cobalt, making them sensitive to the magnetic interaction between tip and sample.

Figure $2 \mathrm{a}$ shows a schematic of attocube system's cantilever-based attoMFM, designed particularly for low temperature and high magnetic field applications. The attoMFM uses a single-mode, fiber-based interferometer [9] to detect tip deflections with noise densities as low as $0.5 \mathrm{pm} / \mathrm{Hz}^{1 / 2}$ [10]. As with most MFMs, the attoMFM applies an AC modulation technique to achieve highest detection sensitivity. In AC mode, the cantilever is mechanically excited at its natural resonance frequency $f_{0}$ using a piezoelectric material oscillating perpendicular to the sample surface. The magnetic interaction offsets the equilibrium position of the tip, which in most cases is hard to detect and therefore ignored. In addition to the pure DC offset, the natural resonance frequency (as well as amplitude and phase) of the cantilever is also affected by the magnetic interaction. This frequency shift $\Delta f=f_{\text {res }}-f_{0}$ can be easily detected by classical lock-in techniques and is the most relevant physical quantity to measure due to its direct proportionality to the local force derivative [11]: $\partial F_{z} / \partial z \sim 2 K \Delta f / f_{0}$. The measurement therefore yields information about the actual local magnetic stray field: $\partial F_{z} / \partial z \sim m_{t i p, z} \partial^{2} H_{z} / \partial z^{2}$ (where $m_{t i p, z}$ is the magnetization of the tip perpendicular to the sample surface) with very high spatial resolution. In a typical MFM measurement, the cantilever is constantly excited at resonance using a $90^{\circ}$ phase-shifted excitation signal. Using this phase-locked loop (PLL) technique, resonance frequency shifts as small as $1 \mu \mathrm{Hz}$ can be detected.

To separate magnetic information from other influences, two techniques are most typically used, referred to as constant height and constant distance mode [12]. In constant height mode, the MFM tip is scanned at a fixed height above the mean sample plane, whereas in constant distance mode the distance between tip and sample is kept precisely constant, compensating any surface corrugation. Constant height mode is typically applied on flat samples, after the sample and scan planes have been aligned parallel. The MFM tip is subsequently retracted by typically $10-100 \mathrm{~nm}$ and is then scanned across the surface with scan speeds of up to several $10 \mu \mathrm{m} / \mathrm{s}$. Any shifts in resonance frequency or phase are recorded simultaneously. This technique is applied to many samples such as hard disks 


\section{LYRA3}

High Resolution Scanning Electron Microscope with Focused Ion Beam for nano-scale imaging, analysis, manipulation and surface modification

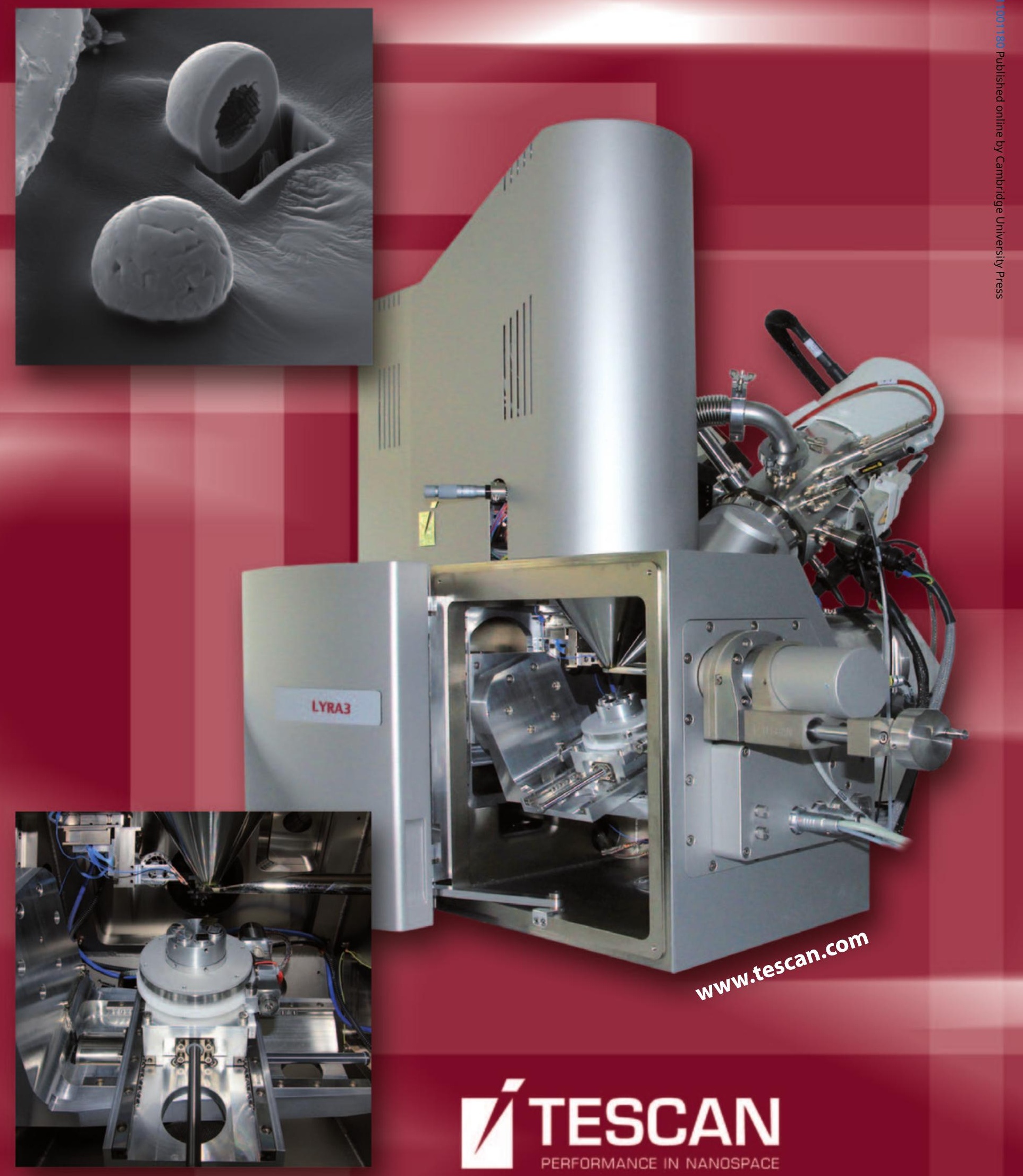


(a)

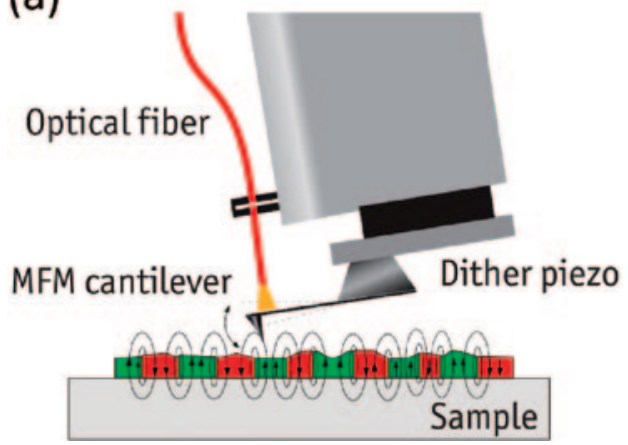

(b)

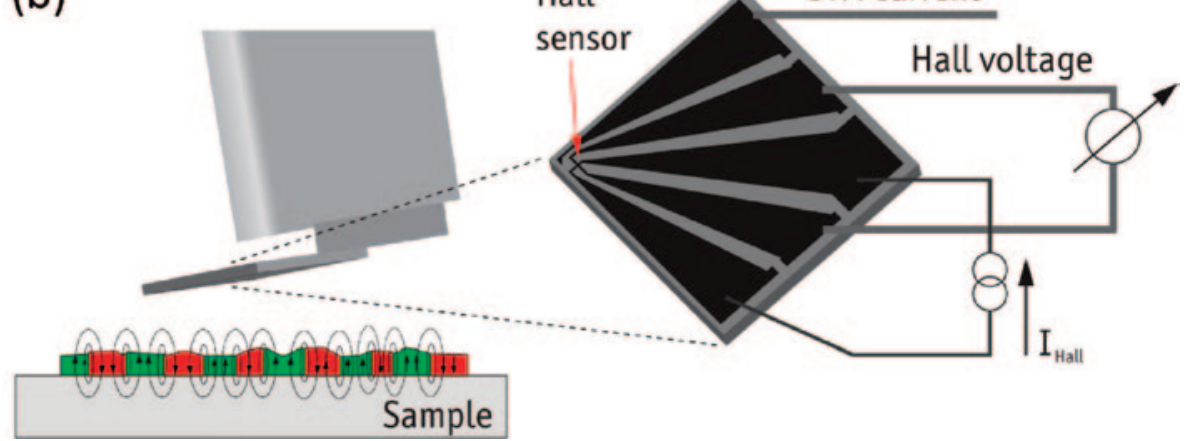

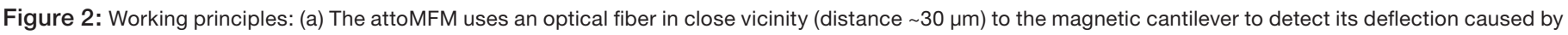

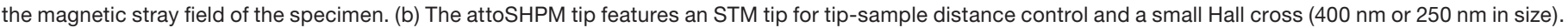

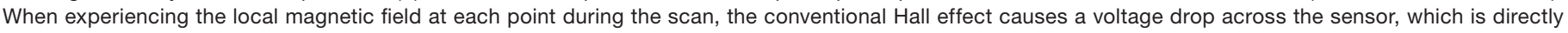
proportional to the magnetic field value.

and superconductors, and it is typically considered preferential because of its ease and speed. In contrast, constant distance mode follows quite exactly the surface topography at a certain separation and is sometimes the only possible way to measure magnetic information of very rough samples. The drawback of constant distance mode is the image acquisition time, which is considerably longer compared to constant height mode.

\section{Scanning Hall Probe Microscopy}

In scanning Hall probe microscopy, a small, typically micron-sized, Hall sensor is scanned in close proximity to the sample surface (see Figure $2 \mathrm{~b}$ ). Mapping the Hall voltage $V_{\mathrm{H}}$ as a function of location directly yields the spatial distribution of the local magnetic field. Similar to MFM, SHPM is most frequently conducted in constant height mode, where the sample plane is typically detected by tunneling current measurements (referred to as STM-tracking SHPM) $[4,13]$.

Today's state-of-the-art Hall sensors are fabricated from silicon or modulation-doped heterostructures using standard CMOS techniques, molecular beam epitaxy, or e-beam lithography. For ultra-high spatial resolution applications, the Hall bar is typically refined by focused-ion beam milling, yielding areal dimensions well below $500 \times 500 \mathrm{~nm}^{2}$.

The figures of merit of Hall sensors are sensitivity and noise. The sensitivity $S_{\text {Hall }}$ of a Hall sensor biased with a current $I$ is given by [14]:

$$
S_{\text {Hall }}=\left|\frac{V_{\mathrm{H}}}{I B}\right| \equiv \frac{1}{e n_{2 \mathrm{D}}},
$$

where $V_{H}$ is the measured Hall voltage, $B$ is the magnetic field experienced by the sensor, $\mathrm{e}=1.6 * 10^{-19}$ [As], and $n_{2 \mathrm{D}}$ is the carrier density in the case of a modulation-doped Hall sensor with a two-dimensional electron gas layer, referred to as 2DEG. Typical values for the sensitivity are 1000-2000 V/AT in a large temperature range [15]. Together with the noise of the sensor, the sensitivity determines the minimal detectable field or field detection limit (DL) of the sensor. There are three sources of noise present in a Hall bar, which are Johnson, $1 / f$, and generationrecombination noise yielding a magnetic DL of the form

$$
\mathrm{DL}=\frac{\sqrt{4 k_{\mathrm{B}} T R \Delta f}}{I S_{\text {Hall }}}+\frac{R \sqrt{\alpha \Delta f}}{\sqrt{N S_{\text {Hall }}}}+\frac{\beta(f) \sqrt{\Delta f}}{N S_{\text {Hall }}}
$$

where $\alpha$ and $\beta$ are (frequency-dependent) proportionality factors, $\Delta f$ is the measurement bandwidth, $I$ the Hall current, $R$ the output resistance, $k_{B}$ the Boltzmann constant, $T$ the sensor temperature, and $N$ the number of charge carriers in the active area of the Hall sensor. From equation (2) it is immediately apparent that larger Hall sensors provide lower $1 / f$ noise because of the larger number of charge carriers present. This typically leads to a lower DL for larger sensors, but this trend disappears at temperatures below $100 \mathrm{~K}$ where heterostructure sensors are typically operated and are dominated by the thermal noise regime. For this temperature range, the magnetic field detection limit is given by

$$
\mathrm{DL}=\frac{\sqrt{4 k_{\mathrm{B}} T R \Delta f}}{I S_{\text {Hall }}} \propto \sqrt{\frac{n_{2 \mathrm{D}}}{\mu}} .
$$

Modern 2DEG Hall sensors such as provided for attocube systems' attoSHPM typically provide carrier mobilities larger than $160,000 \mathrm{~cm}^{2} / \mathrm{Vs}$ at densities of $4 \times 10^{11} \mathrm{~cm}^{-2}$, yielding detection limits of $15 \mathrm{nT} / \mathrm{Hz}^{1 / 2}$ at $4 \mathrm{~K}$ and $40 \mu \mathrm{A}$ excitation current. In real life, however, the practical attainable field detection limit has so far been limited to the $\mu \mathrm{T}$ range in the few Hertz bandwidth for most experiments [16]. This is due to current fluctuations in the current source, which are directly translated into voltage fluctuations because of the intrinsic voltage offsets in the Hall bar.

\section{Examples and Applications}

Vortices in superconductors. An example of the quantum objects that are intensely studied with these methods are vortices in superconductors, motivated in large part by the desire to pin down the nature of high- $\mathrm{T}_{\mathrm{c}}$ superconductivity, which was discovered in 1986 [17]. The superconducting state forms below limiting values of temperature $\left(T_{c}\right)$, magnetic field $\left(H_{c}\right)$, and electrical current $\left(j_{c}\right)$. Superconducting materials possess two distinct outstanding properties: electrical currents (up to $j_{c}$ ) are carried with virtually no electrical resistance, and external magnetic fields are completely expelled from the material's interior (ideal diamagnetism). Most superconductors show an intermediate state above the lower critical magnetic field $H_{c 1}$, at which magnetic flux is allowed to enter the specimen in the form of circular supercurrents, each containing exactly one magnetic flux quantum $\left(\Phi_{0}=2.07 * 10^{-15} \mathrm{Tm}^{2}\right)$. 
These quantum objects are referred to as vortices and can be considered single entities. The density of vortices can be tuned and is directly proportional to the applied external magnetic field-as long as the applied external magnetic field remains below the critical field $H_{c 2}$, where the sample becomes normal conducting. Through the mutual repulsion of neighboring circular currents, a vortex lattice forms, which in the easiest case is hexagonal [18]. In addition to the mutual repulsion between vortices, pinning forces are present to a variable extent in every superconducting material. These forces cause vortices to stick to certain locations on the surface or in the bulk of the superconductor. This behavior has important implications for some applications like the construction of superconducting magnets: upon application of an electrical current, vortices experience a Lorentz force, which causes them to move. Vortex motion, however, induces a voltage and thus electrical resistance, which is not desired in such coils. Hence, (artificial) vortex pinning plays an important role in minimizing electrical losses due to moving vortices.

The vortex lattice can easily be observed on a freshly cleaved $\mathrm{Bi}_{2} \mathrm{Sr}_{2} \mathrm{CaCu}_{2} \mathrm{O}_{8+\mathrm{x}}(\mathrm{Bi}-2212)$ cuprate superconductor, as imaged using an attoMFM in Figure 3 (left). The observed lattice is almost perfectly hexagonal, indicating that hardly any pinning is present in this compound. With an inter-vortex spacing $d$ of $700 \pm 34 \mathrm{~nm}$, the vortex separation is in good agreement with the expected value of $(4 / 3)^{1 / 4}\left(\phi_{0} / H\right)^{1 / 2}=729 \mathrm{~nm}$ for an external magnetic field of 45 gauss.

Vortices and vortex lattices have been intensively studied in Bi-2212, YBCO, and many other cuprate compounds [19]. This effort has significantly improved the understanding of the different vortex types, phases, and pinning mechanism existing, but it has not solved the mystery of the origin of high- $T_{c}$ superconductivity.

The recently discovered new class of iron-arsenic based high- $T_{c}$ superconductors (pnictides) [20] gave new spirit to solving the puzzle of high- $T_{c}$ superconductivity. One of the first MFM images recorded on the pnictide compound $\mathrm{Ba}_{1-\mathrm{x}} \mathrm{K}_{\mathrm{x}} \mathrm{Fe}_{2} \mathrm{As}_{2}$ was demonstrated by attocube systems in 2009 and is shown in Figure 3 (right). In contrast to the Bi-2212, the pnictide sample shows strong pinning leading to a significantly disordered vortex lattice. Independent investigations on the related compound $\mathrm{BaFe}_{1.8} \mathrm{Co}_{0.2} \mathrm{As}_{2}$ confirm these results
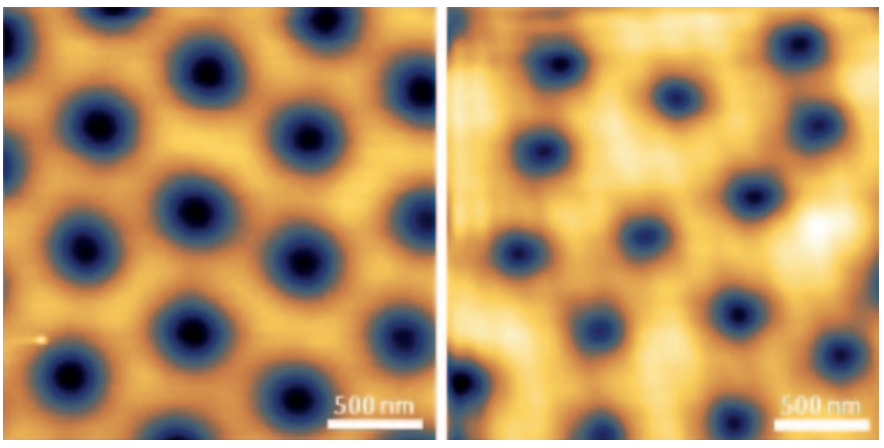

Figure 3: Vortex images recorded using low-temperature MFM on the cuprate $\mathrm{Bi}_{2} \mathrm{Sr}_{2} \mathrm{CaCu}_{2} \mathrm{O}_{8+x}$ (left, attocube applications labs, 2009; sample courtesy of A. Erb, TU Munich) and the pnicitide $\mathrm{Ba}_{1-\mathrm{x}} \mathrm{K}_{\mathrm{x}} \mathrm{Fe}_{2} \mathrm{As}_{2}$ (right, attocube applications labs, 2009; sample courtesy of H.H. Wen, Beijing), respectively. Measurements were conducted at $4.1 \mathrm{~K}, 45$ gauss, and $70 \mathrm{~nm}$ tip-sample distance in both cases. Dark-bright contrast is approximately $200 \mathrm{mHz}$ in both cases. and relate the observed pinning to bulk pinning rather than surface pinning [21], which may enable further tailoring of the properties of these new materials to even higher critical currents as desired for applications such as wiring for superconducting ultra-high-field magnets or power grids.

In contrast to many other magnetic imaging techniques, MFM can also be used for local manipulation of samples: in 2009 O. Auslaender et al. managed to individually drag single vortices, thus being able to directly probe the interaction with the local disorder potential [22]. Whereas previous experiments usually yielded information on the properties of bulk pinning as experienced by large ensembles of vortices, this approach allowed control of the properties of vortex matter on a local scale.

Data storage. Another field of research with high application potential for MFM is data storage and the accompanying material science. Although market demands for high disc capacity have already been addressed by altering hard disc magnetization orientation from longitudinal to perpendicular, further means of increasing storage density are under consideration. One of the most promising candidates, with storage densities of $1 \mathrm{Tbit} / \mathrm{in}^{2}$ and beyond, is bit-patterned media (BPM), where single domain particles are defined by lithography or self-assembly. BPM eliminates the random noise associated with multi-grain bits, defines sharper transitions between bits, and overcomes the problem of poor thermal stability compared to conventional recording media [7]. MFM proves to be an ideal tool for the characterization of such materials in cryogenic conditions, where high magnetic fields are readily available and magnetic switching and hysteresis of BPM can be investigated free of thermal effects (see Figure 4).

In contrast to MFM, SHPM is most frequently used in applications where quantitative information on local magnetic properties is required. Typical applications are local hysteresis and domain structure measurements in ferromagnetic and multiferroic materials [23], as well as flux penetration studies [13] in superconductors. The latter experiment is depicted in the upper part of Figure 5, where the flux distribution on a degraded $\mathrm{Bi}_{2} \mathrm{Sr}_{2} \mathrm{CaCu}_{2} \mathrm{O}_{8+\mathrm{x}}$ surface is measured. The emergent field of the vortex can be modeled within the framework of the London approximation, yielding penetration depth $\lambda$
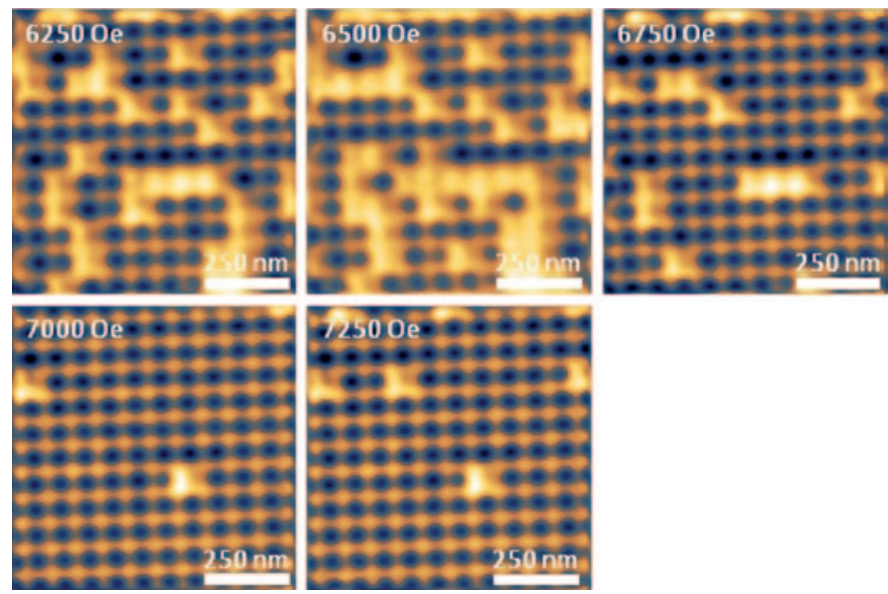

Figure 4: Bit patterned media sample: Magnetic domain switching upon externally applied magnetic field, measured at $4.2 \mathrm{~K}$. Dark-bright contrast is approximately $6.2 \mathrm{~Hz}$ for all measurements (sample courtesy of E. Fullerton, Hitachi [USA]). 

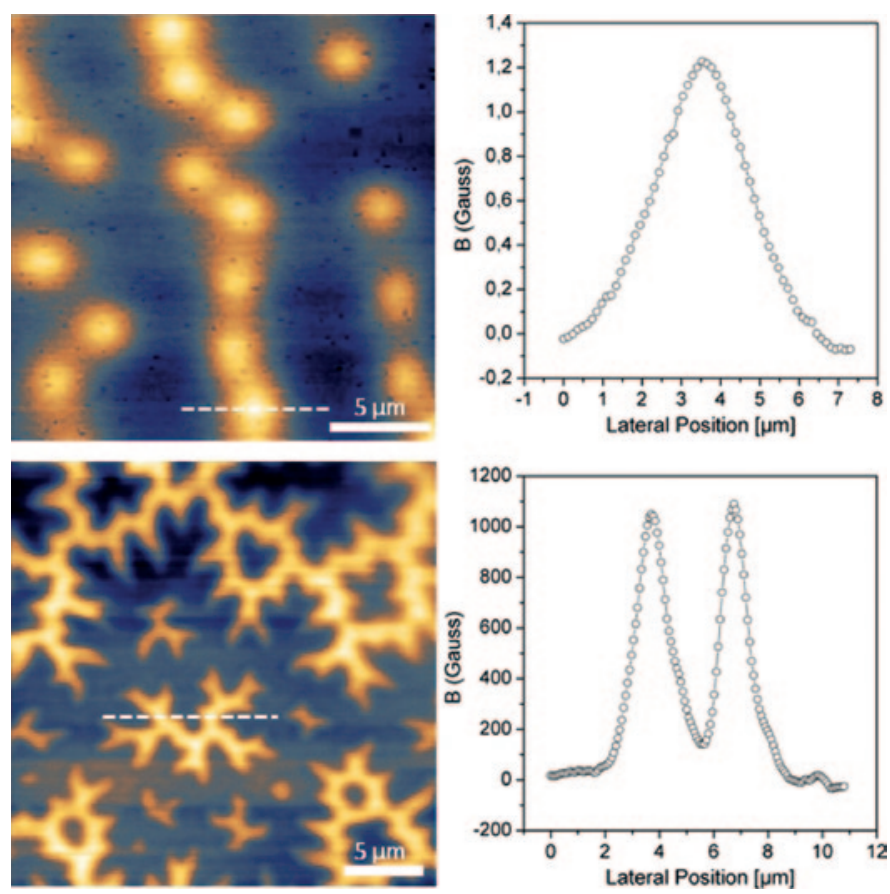

Figure 5: Upper part: SHPM measurements on a degraded $\mathrm{Bi}_{2} \mathrm{Sr}_{2} \mathrm{CaCu}_{2} \mathrm{O}_{8+\mathrm{x}}$ substrate showing strong surface pinning effects at $4.2 \mathrm{~K}$ and 2.5 gauss magnetic field. The figure to the right shows a linecut through one of the vortices, displaying the field distribution approximately $100 \mathrm{~nm}$ above the surface (attocube applications lab, 2011; sample courtesy of A. Erb, TU Munich). Lower part: SHPM measurement of a BaFeO ferromagnet, measured at $300 \mathrm{~K}$. The figure to the right shows a linecut through one of the magnetic domains, indicating the strong magnetization of the ferromagnet (attocube applications labs, 2011; sample courtesy of R. Kramer, Grenoble).

and coherence length $\xi$, respectively [13]. Another example for quantitative field distribution measurements is given in the lower part of Figure 5, depicting ferromagnetic domains in the compound barium hexaferrite: it shows extremely strong magnetization (see the right hand side of Figure 5) and is currently investigated in the course of the search for replacements for rare earth materials [24].

Improved cooling technology. From a different perspective, low-temperature research on magnetic nanostructures faces yet another challenge-liquid helium shortage and the corresponding increase in operation costs [25]. This leads to the demand for cryogen-free cooling systems. Sensitive techniques such as MFM and SHPM, however, require specially designed products optimized for ultra-low vibration levels. Combining the latter with high magnetic fields has become possible only very recently because of a proprietary (top-loading) design by attocube systems: mechanical vibrations created by a pulse-tube coldhead are decoupled from the measurement platform, resulting in peak-to-peak vibration amplitudes of less than $4.2 \mathrm{~nm}$ at the sample location while retaining probe cooldown times as fast as 1 hour to $4 \mathrm{~K}$ [26]. This technology can be extended to temperatures as low as $50 \mathrm{mK}$, as recently demonstrated [27].

\section{Summary}

It has been shown that low temperature MFM and SHPM play a crucial role in many fields of fundamental research. With a large number of high-impact customer publications and a company history of more than a decade, attocube systems is a

prime supplier of scanning probe microscope instruments and corresponding cooling systems. This company is spearheading the development of ultra-low vibration, cryogen-free cooling equipment, enabling application of MFM and SHPM at temperatures as low as $50 \mathrm{mK}$ without the need for costly liquid helium or nitrogen.

\section{Acknowledgments}

We would like to thank our colleagues H. Qian, C. Debuschewitz, and R. Pohlner for assistance with some of the measurements, and Simon Bending for useful discussions. A special thanks goes also to A. Erb, H.H. Wen, R. Kramer, and E. Fullerton for providing samples.

\section{References}

[1] VV Volkov and Y Zhu, Ultramicroscopy 98 (2004) 271-81.

[2] P Weinberger, Phil Mag Lett 88 (2008) 897.

[3] Y Martin and HK Wickramasinghe, Appl Phys Lett 50 (1987) 1455; and JJ Saenz et al., J Appl Phys 62 (1987) 4293.

[4] AM Chang et al ., Appl Phys Lett 61 (1992) 1974.

[5] J Paglione and RL Greene, Nature Physics 6 (2010) 645.

[6] I Žutić et al., Rev Mod Phys 76 (2004) 323-410.

[7] R New, "The Future of Magnetic Recording Technology," Hitachi Global Storage Technologies, 2008.

[8] G Binnig, CF Quate, and C Gerber, Phys Rev Lett 56 (1986) 930.

[9] SJ Bending, Adv Phys 48 (1999) 449, and private communication with SJ Bending (2011).

[10] D Rugar, HJ Mamin, and P Guethner, Appl Phys Lett 55 (1989) 2588.

[11] L Abelmann et al., "Magnetic Force MicroscopyTowards Higher Resolution" in Magnetic Microscopy of Nanostructures, eds. H Hopster, HP Oepen, Springer, Berlin, 2005; Y Zhu, "Chapter 11: Magnetic force microscopy", 411-451, in Modern Techniques for Characterizing Magnetic Materials, ed. Y Zhu, Springer, New York, 2005.

[12] K Babcock et al., Mat Res Soc Symp 355 (1995) 311.

[13] VV Khotkevych, MV Milošević, and SJ Bending, Rev Sci Instrum 79 (2008) 123708.

[14] RS Popović, Hall Effect Devices, ed. A Hilger, Bristol, UK, 1990.

[15] K Vervaeke, E Simon, G Borghs, and VV Moshchalkov, Rev Sci Instrum 80 (2009) 074701.

[16] P Kejik et al., Sens Actuators, A 129 (2006) 212.

[17] JG Bednorz and KA Mueller, $Z$ Phys B-Condensed Matter 64 (1986) 189.

[18] AA Abrikosov, Zh Eksp i Teor Fiz 32, (1957) 1442; Soviet Phys JETP 5 (1957) 1174.

[19] Takahashi et al., Nature 453 (2008) 376.

[20] Y Kamihara, T Watanabe, M Hirano, and H Hosono, J Am Chem Soc 130 (2008) 3296.

[21] Y Yin et al., Phys Rev Lett 102 (2009) 097002.

[22] O Auslaender et al., Nature Physics 5 (2009) 35; Reichhardt, Nature Physics 5 (2009) 15.

[23] H Schmid, Ferroelectrics 162 (1994) 317.

[24] F Mazaleyrat et al., arXiv:cond-mat/1103.5840.

[25] KH Kaplan, Physics Today (June 2007) 31.

[26] http://www.attocube.com/attoCRYO/attoDRY1000.htm

[27] http://www.attocube.com/attoCRYO/attoDRY5000.htm 
